\title{
Uninephrectomy of Diabetic OVE26 Mice Greatly Accelerates Albuminuria, Fibrosis, Inflammatory Cell Infiltration and Changes in Gene Expression
}

\author{
Shirong Zheng ${ }^{a, b}$ Yun Huang ${ }^{a}$ Lu Yang $^{c}$ Teresa Chen ${ }^{c}$ Jianxiang $\mathrm{Xu}^{\mathrm{a}}$ \\ Paul N. Epstein ${ }^{a}$ \\ a Department of Pediatrics, ${ }^{b}$ Department of Surgery, Institute for Cellular Therapeutics, and ${ }^{\mathrm{C}}$ Department of \\ Pharmacology and Toxicology, University of Louisville, Louisville, Ky., USA
}

\section{Key Words}

Albuminuria $\cdot$ Interstitial fibrosis $\cdot$ Microarray $\cdot$ Diabetic

nephropathy $\cdot$ Inflammation

\begin{abstract}
Background/Aims: OVE26 (OVE) mice provide a valuable model of advanced diabetic nephropathy (DN), but they take 8 months to develop moderate interstitial fibrosis and reduced glomerular filtration rate (GFR). The aim of this project was to produce a more rapid and advanced model of DN. Methods: Uninephrectomy was applied to OVE and FVB mice at 2 months of age. Albuminuria, GFR, glomerulosclerosis, interstitial fibrosis, gene expression and monocyte infiltration were evaluated as a function of diabetes and uninephrectomy. Results: Albuminuria, monocyte infiltration, mesangial matrix expansion and renal fibrosis were greatly accelerated in uninephrectomized mice. DN was more advanced 10 weeks after uninephrectomy than in untreated OVE mice at 8 months of age. Uninephrectomy had almost no effect on these characteristics in non-diabetic mice. Microarray studies indicated that the accelerated fibrosis and cell infiltration in nephrectomized OVE mice were accompanied by corresponding gene expression changes in canonical pathways for fibrosis and inflammation. Conclusion: Uninephrectomy greatly accelerates all features of diabetic re-
\end{abstract}

nal damage. This procedure provides a 10-week period after surgery to examine very large changes in the pathology of DN. The model may be particularly useful for testing new therapies and for analysis of the progression of albuminuria and fibrosis in DN.

Copyright $\odot 2011$ S. Karger AG, Basel

\section{Introduction}

Diabetes is the major cause of end-stage renal diabetes worldwide. Our understanding of the mechanism and treatment of diabetic nephropathy $(\mathrm{DN})$ are limited by the lack of reliable animal models that mimic advanced human disease. Major efforts have been made to modify existing models or develop new models to accelerate the progression of DN. Attention has focused on mouse models since mice provide the most convenient mammal for genetic manipulation which is invaluable for studying molecular pathology and signal transduction. Although advances have been made by identifying the most susceptible mouse strains $[1,2]$ and by producing genetic modifications, such as endothelial nitric oxide synthase deficiency $[3,4]$, there is still no single mouse model that reproduces all features of advanced human DN [5].

\section{KARGER}

(C) 2011 S. Karger AG, Basel

Fax +4161306 1234

E-Mail karger@karger.ch

www.karger.com
Accessible online at:

www.karger.com/nee
Paul N. Epstein

University of Louisville, 570 S. Preston St.

Louisville, KY 40202 (USA)

Tel. +1 5028522655

E-Mail paul.epstein@louisville.edu 
OVE26 (OVE) mice develop early-onset, insulinopenic diabetes due to a transgene regulated by the rat insulin 2 promoter that overexpresses calmodulin in pancreatic $\beta$-cells. They provide a useful model of advanced DN with respect to albuminuria and other pathological features including glomerular mesangial matrix expansion and podocyte damage $[2,5-7]$. However, even modest interstitial fibrosis takes 5-8 months to develop and a $20 \%$ decline in glomerular filtration rate (GFR) does not occur until 9 months of age [6]. The severity of OVE DN progresses with age but this approach is limited by high death rates in older OVE mice. To speed the progression of advanced DN, we are testing removal of one kidney from OVE mice. Uninephrectomy has been used in other models to accelerate nephropathy [8-10]. In this study we show that characteristics of DN, i.e. albuminuria, interstitial fibrosis, glomerulosclerosis, changes in GFR, and abnormal gene expression that normally take 8-9 months to develop, are more advanced in uninephrectomized OVE mice within 10 weeks of surgery, at 4.5 months of age. Nephrectomized OVE mice are a much faster alternative model for studying advanced renal disease in diabetes.

\section{Materials and Methods}

\section{Animals}

All animal procedures in this study followed the NIH Guide for the Care and Use of Laboratory Animals and were approved by the University of Louisville Institutional Animal Care and Use Committee. OVE diabetic mice on the FVB background were bred in our laboratory and FVB breeder mice were obtained from Charles River (Charles River Laboratories International, Inc. Wilmington, Mass., USA). All animals had free access to standard rodent chow and water throughout the study.

\section{Uninephrectomy}

Female OVE and littermate FVB mice were used in the study. OVE and FVB mice were randomly assigned into uninephrectomy (uni) or sham procedures which divided the mice into 4 groups of 8 mice: OVE-uni, OVE-sham, FVB-uni, and FVB-sham. At 2 months of age, uninephrectomy and sham surgeries were performed under ketamine/xylazine anesthesia. For uninephrectomy, the left kidney was surgically removed via a left paramedian incision on the back. The adrenal gland was carefully freed from the upper pole of the renal capsule before the renal pedicle was ligated and the kidney removed, the incision was closed with sutures. For sham surgery, the kidney was manipulated without ablation. Cefazolin was injected subcutaneously, $400 \mu \mathrm{g}$, twice a day, for 2 days to prevent infection. Buprenorphine was used after surgery for pain control. All mice were sacrificed under anesthesia at 4.5 months of age, which was 10 weeks after surgery. Prior to harvest of the remnant kidney, mice were perfused with phosphate-buffered saline. Half of the kidney from each animal was snap frozen in liquid nitrogen and stored at $-80^{\circ} \mathrm{C}$ for RNA and protein extraction. The other half was fixed with formalin for histology.

\section{Measurement of Urinary Albumin Excretion GFR and Blood} Glucose

For 24-hour urine collection, individual mice were placed in metabolic cages with access to chow and 10\% liquid diet (Glucerna, Abbott Laboratories), as we have described previously [6]. Urinary albumin concentration was measured with a mouse albumin ELISA kit (Bethyl Laboratories, Montgomery, Tex., USA) within the linear range of the assay and expressed as $\mu \mathrm{g} / 24 \mathrm{~h}$. GFR was determined from creatinine in 24-hour urine samples and plasma samples obtained from tail blood. Plasma and urine creatinine concentrations were measured by HPLC with electrochemical detection $[11,12]$. GFR was calculated with the formula: $\mathrm{GFR}=$ (total creatinine $\mu \mathrm{g}$ from $24 \mathrm{~h}$ urine $) /($ plasma creatinine concentration $\mu \mathrm{g} / \mu \mathrm{l}) /(24 \mathrm{~h} \times 60 \mathrm{~min} / \mathrm{h})$. Glucose was measured in serum samples obtained from tail blood of non-fasted mice. Glucose was assayed by the hexokinase kit (Sigma-Aldrich).

\section{Assessment of Renal Pathology}

Kidneys were fixed overnight in $10 \%$ neutral buffered formalin and embedded in paraffin. Tissue sections were cut at $3 \mu \mathrm{m}$ and stained for hematoxylin and eosin, periodic acid-Schiff (PAS) and Masson's trichrome using standard protocols. Glomerular volume, mesangial fraction and mesangial volume were determined from PAS-stained sections. Digital images of random glomeruli were obtained at $400 \times$ by an observer blind to the identity of the section. These were then analyzed by a second blind observer to determine the cross-sectional area of the glomerular tuft and the mesangial area. The cross-sectional area of the glomerular tuft (AG) was determined from outlines of the tuft using the program Adobe Photoshop. Glomerular volume (VG) was calculated from the cross-sectional area with the formula $\mathrm{VG}=\beta / \mathrm{k}$ $(A G)^{3 / 2}$, where $\beta=1.38$ is the shape coefficient for a sphere and $\mathrm{k}=1.1$ is the size distribution coefficient $[13,14]$. The mesangial area $(\mathrm{AM})$ within the glomerular tuft was defined as the area that stained positively for PAS $[15,16]$. Positive staining was identified using the capacity of Adobe Photoshop to select areas of matching color intensity. Mesangial fraction (Mf) was calculated as the ratio, AM/AG, and mesangial volume (VM) was calculated as the product, $\mathrm{Mf} \times \mathrm{VG}$. Four mice were analyzed per group and 15 glomeruli were measured per mouse.

Renal fibrosis was assessed by immunohistochemical staining with rabbit anti-collagen I antibody (Abcam Inc., Cambridge, Mass., USA). Fibrosis scores were expressed as the number of pixels stained for collagen per $200 \times$ magnification image field. The digital images were obtained by an observer blind to the identity of the section and quantified by another blind observer to measure the number of positive pixels. Staining around large vessels was excluded from the analysis. Pixel number was determined using the capacity of Adobe Photoshop to select areas of matching color intensity. Six randomly selected non-overlapping fields from the cortical region were obtained per kidney section and 3-5 mice were included in each group.

Infiltrating monocytes were identified using the $\alpha$-napthyl acetate esterase histochemical kit (Sigma-Aldrich) following the manufacturer's instructions. Staining was rated by a blind observer using the following scoring system: $0=$ no stained cells, 
$1=$ rare stained cells, $2=$ occasional clusters of 5 or fewer stained cells, $3=$ more than 3 clusters of at least 5 stained cells.

\section{Microarray Hybridization and Gene Expression Analysis}

RNA was extracted from frozen kidney using TRIzol reagent (Invitrogen, Carlsbad, Calif., USA). RNA integrity was confirmed using an Agilent 2100 bioanalyzer. RNA was pooled from 4 mice in each group (OVE-uni, OVE-sham, FVB-uni, FVB-sham). A 100 -ng aliquot of RNA from the pooled sample was used for probe preparation. Preparation of cDNA was carried out using the onecycle cDNA synthesis kit with oligo-dT-T7 promoter primers (Affymetrix, Inc., Santa Clara, Calif., USA). The cDNA served as template for T7 RNA polymerase transcription in the presence of biotin-labeled CTP and UTP. The cRNA was fragmented to a size range of 50-200 nucleotides. Probes were hybridized to Affymetrix MG-430 2.0 GeneChips and scanned by GCS 3000 7G scanner and signals analyzed with GCOS software (Affymetrix, Inc.). To identify genes altered by diabetes, two comparisons of signal intensity were made, OVE-uni versus FVB-uni and OVE-sham versus FVBsham. The ratio of normalized signal intensity was determined for each probe set on the chip. Genes with a 2 -fold or greater change in signal were uploaded to Ingenuity software (Ingenuity Pathway Analysis, Redwood City, Calif., USA) for pathway analysis.

\section{Quantitative Reverse Transcriptase PCR}

The same RNA samples used for microarray assay prior to pooling were used for rtPCR. The cDNA was synthesized with a high-capacity cDNA archive kit (p/n 4322171; Applied Biosystems, Foster City, Calif., USA) and PCR was performed on an Applied Biosystems 7300 thermocycler using commercially available Taqman reagents (Assay on Demand; Applied Biosystems) for C3 (Mm00437858-m1), CCL5 (Mm01302428ml), TIMP1 (Mm01341361-ml), TIMP3 (Mm00441827-ml), MMP3 (Mm01168713-ml), Nephrin: Mm-00497828_m1, Collagen 1: Mm01302043_g1, Collagen 3: Mm 01254476_m1, and VCAM1 (mM01320970-ml). Amplification was performed in duplicate using 40 cycles of denaturation at $95^{\circ} \mathrm{C}$ for $15 \mathrm{~s}$, primer annealing/extension at $60^{\circ} \mathrm{C}$ for $1 \mathrm{~min}$. Expression data were normalized to 18s ribosomal RNA (Hs99999901-sl) measured for the same samples. The relative expression ratio was calculated according to the $2^{-\Delta \Delta C T}$ method.

\section{Statistical Analyses}

Data are expressed as means \pm SE. Comparison between groups was performed by one- or two-way ANOVA. Correlations between gene expression ratios of different groups were determined by the Pearson product moment correlation coefficient. Statistical analyses were performed with SigmaStat software.

\section{Results}

Uninephrectomy performed on 2-month-old OVE and FVB littermates did not result in abscess formation at the surgical site. The acute death rate from anesthesia or bleeding during surgery was less than $10 \%$ and the death rate up to 10 weeks was $30 \%$. Uninephrectomy did not significantly alter blood glucose of diabetic mice (fig. 1a). Body weight of OVE and FVB mice tended to be lower after uninephrectomy than in sham treated mice but this difference did not reach statistical significance during the 10-week observation period (fig. 1b). Uninephrectomy significantly increased the weight of the remnant kidney, especially in diabetic mice (fig. 1c). As shown in figure 1d, urine volume was much higher in OVE mice than in FVB mice but urine volume was not altered by uninephrectomy in the diabetic or control groups.

\section{Albuminuria and GFR}

As shown in figure 2, uninephrectomy produced a striking increase in albuminuria of OVE mice but had no significant effect on albumin excretion of FVB mice. Albuminuria increased within 2 weeks of surgery and continued to increase through 10 weeks post-surgery. At this time point, albuminuria of OVE-uni mice was 600 -fold higher than in non-diabetic mice, and more than 6 times higher than that of OVE-sham mice.

GFR estimated by creatinine clearance is shown in figure 3. In FVB mice, uninephrectomy had no effect on GFR. Prior to surgery, GFR was already higher in diabetic mice than in FVB mice. For OVE-sham mice, GFR remained high throughout the 10 weeks of observation, but in OVE-uni mice GFR appeared to be declining by 10 weeks of age. The decline in GFR was statistically significant and evident in all 4 OVE-uni mice assayed at 4 and 10 weeks post-surgery (fig. $3 \mathrm{~b}$ ). In the OVE-sham group, the change in GFR varied among mice during the same time period (fig. 3c).

\section{Morphology, Interstitial Fibrosis and Monocyte \\ Infiltration}

Uninephrectomy markedly increased glomerulosclerosis in OVE-uni mice assessed 10 weeks after surgery (fig. 4). In contrast, FVB mice showed a minimal effect of uninephrectomy on glomerular morphology. The effect of uninephrectomy to increase glomerular size and mesangial matrix content in OVE mice was evident by trichrome and PAS staining, and this impression was confirmed by quantitative analysis of PAS-stained sections (fig. 4i-k). Glomerulosclerosis became more severe following uninephrectomy of OVE mice. Segmental glomerulosclerosis and circumferential fibrous crescents, recognized by acellular, eosinophilic nodules inside the tuft and PAS-positive fibers surrounding Bowman's capsule, were more obvious in OVE-uni mice (fig. 4h) than in OVE-sham mice (fig. 4g). In addition to its effects on morphology, uninephrectomy also altered expression of the glomerular podocyte gene, nephrin (fig. 4l). 

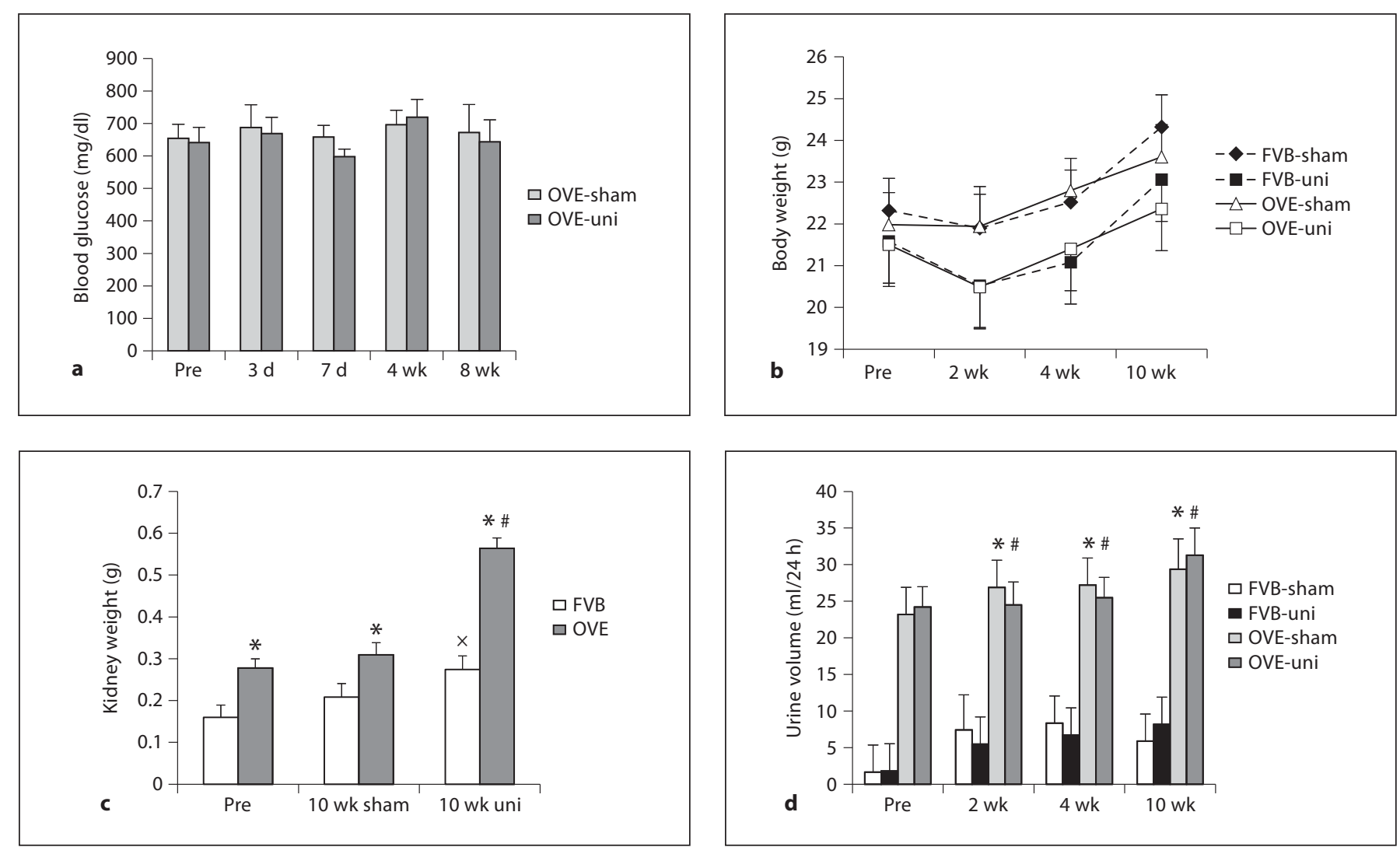

Fig. 1. Effects of uninephrectomy on blood glucose (a), body weight (b), kidney weight (c) and urine volume (d) in diabetic OVE mice. Blood glucose (a), measured in 4-5 non-fasting diabetic mice per group, was not affected by uninephrectomy. Uninephrectomy did not significantly change body weight (b) in either diabetic or non-diabetic mice. Kidney weights (c) were higher in diabetic mice than FVB mice (indicated by ${ }^{*} \mathrm{p}<0.05$ ) and uninephrectomy increased kidney weights in OVE-uni mice compared to OVE-sham mice $(\# \mathrm{p}<0.05)$ and in FVB-uni mice

Fig. 2. Uninephrectomy (uni) increases albuminuria in OVE mice but not in FVB mice. 24-hour urine albumin was measured before and after uni or sham surgery. Values were converted to $\log _{10}$ to put all values on the graph. * indicates that OVE-uni values were greater than OVE-sham at the same time point $(\mathrm{p}<0.01)$ and that albuminuria increased at each subsequent time point after uninephrectomy in OVE mice $(\mathrm{p}<0.01) .{ }^{\#}$ indicates that albuminuria in OVE-sham mice increased from 4 to 10 weeks after sham surgery. In all instances, OVE values were greater than FVB values $(\mathrm{p}<0.01) . \mathrm{n}=5-8$ for each group. Comparisons were performed by two-way ANOVA.

compared to FVB-sham mice $\left({ }^{\times} \mathrm{p}<0.05\right)$. Uninephrectomy did not change the urine volume (d) in either diabetic mice or nondiabetic mice. OVE mice always produced much more urine than FVB mice. In panel $\mathbf{d}^{*}$ or ${ }^{*}$ indicates that the urine volume in OVE-sham and OVE-uni mice was significantly higher than in FVB-sham or FVB-uni mice at all time points $(\mathrm{p}<0.05) . \mathrm{n}=$ 5-9 for each group. Comparisons were performed by two-way ANOVA.

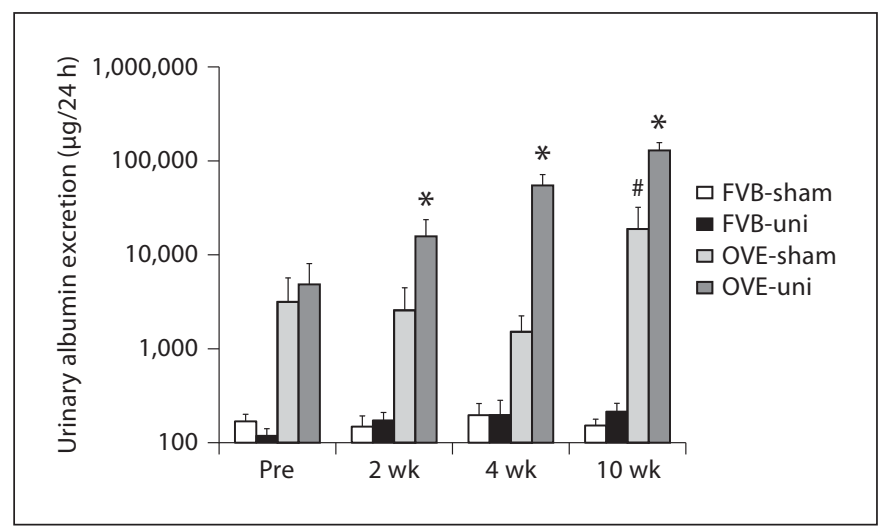




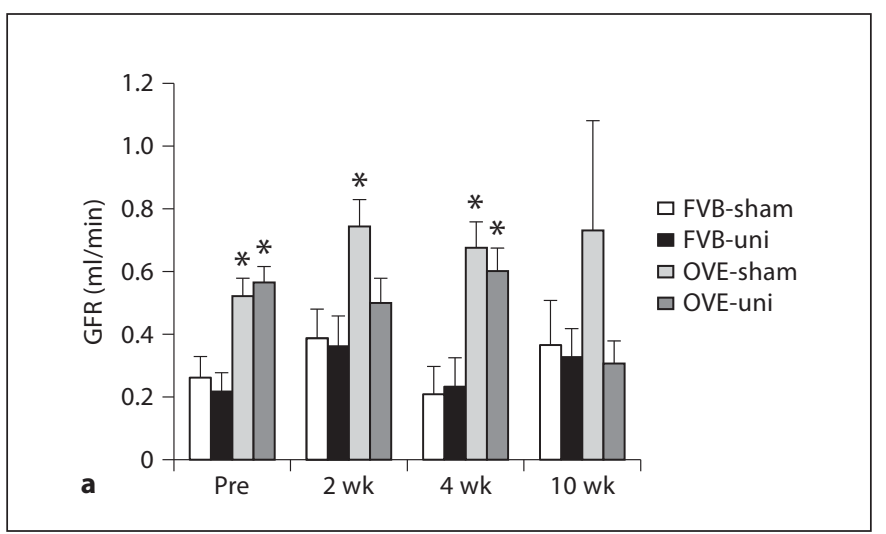

Fig. 3. Effect of diabetes and uninephrectomy (uni) on GFR. a GFR determined by creatinine clearance and HPLC before and 2, 4 and 10 weeks after surgery. ${ }^{*}$ indicates that each OVE group was higher than the corresponding FVB group at the same time

Uninephrectomy significantly reduced nephrin expression in FVB mice. OVE mice already have reduced nephrin expression [6] compared to FVB mice and uninephrectomy resulted in a non-significant trend towards lower nephrin expression in OVE mice $(\mathrm{p}=0.12)$.

Uninephrectomy of OVE mice increased damage in tubular and interstitial areas. Aggravated tubular damage was evident as more severe tubular dilation with tubular cell atrophy and more frequent protein casts (fig. $4 \mathrm{~d}$, h). Immunohistochemical staining for collagen I (fig. 5ad) demonstrated a dramatic increase in fibrosis in the remnant kidney of OVE-uni mice. Quantitation of collagen I staining (fig. 5e) indicated that uninephrectomy produced a 4 -fold increase in the area of collagen I deposition in OVE renal cortex. Correlation analysis between the area of collagen I staining and albumin excretion rate showed a significant association between renal fibrosis and the severity of albuminuria (fig. 5f). Consistent with the increase in renal fibrosis, we also found that uninephrectomy produced a significant increase in the expression of mRNAs for collagen 1 and collagen 3 (fig. 5g, h).

Sections from 4 or 5 mice per group were stained by reaction of endogenous esterases with the substrate $\alpha$ napthyl acetate, a histochemical marker of infiltrating monocytes. Stained sections were scored from 0 to 3 by the rating system described in the Methods section. As shown in figure 6, no infiltrating cells were observed in FVB-sham or FVB-uni mice. Only 1 OVE-sham mouse had infiltrating monocytes but all 5 OVE-uni mice had moderate or extensive infiltration.

Uninephrectomy and OVE26 Diabetic Nephropathy

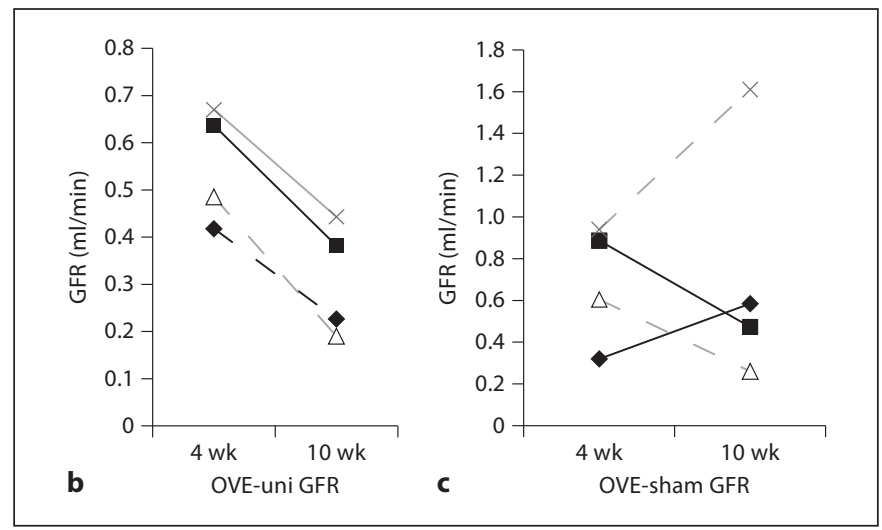

point $(\mathrm{p}<0.02)$. $\mathbf{b}$ GFR declined in each of 4 OVE-uni mice from 4 to 10 weeks after surgery ( $<<0.01$ by paired test). c During the same time period, GFR varied up and down in OVE-sham mice.

\section{Gene Expression Profiles}

Pooled RNA from 4 mice of each group was used to prepare probes that were hybridized to Affymetrix MG4302.0 GeneChips. To identify genes altered by diabetes, two comparisons of signal intensity were made, OVE-uni versus FVB-uni (designated as UNI ratio) and OVEsham versus FVB-sham (designated as SHAM ratio). Since the use of pooled samples precluded statistical comparisons between groups, we also performed rtPCR on 9 genes to assess the reliability of the gene array results. Table 1 shows the UNI ratios and SHAM ratios for 9 genes tested by both gene array and by rtPCR. Of 17 comparisons that can be made between ratios obtained by rtPCR and by gene array, 13 show similar ratios by both methods. In addition, for the 11 statistically significant rtPCR results, 10 of the ratios obtained by gene array were similar.

The comparison of signal intensity of OVE-uni versus FVB-uni revealed 1,968 UNI genes that were increased or decreased at least 2 -fold by diabetes. The comparison of OVE-sham versus FVB-sham revealed 1,615 SHAM genes increased or reduced at least 2 -fold by diabetes. These genes were uploaded into the Ingenuity Pathway Analysis program. Table 2 lists the 15 most significant canonical pathways found by Ingenuity in the UNI dataset. The hepatic fibrosis/stellate activation pathway was the top canonical pathway. This pathway contains many genes that are involved in renal fibrosis. The genes showing diabetes induced changes in the UNI dataset included collagen (COL1A1, COL1A2 and COL3A1) and other matrix proteins such as fibronectin (FN1) and laminin

Nephron Exp Nephrol 2011;119:e21-e32 e25 



Fig. 4. Uninephrectomy effects on OVE glomeruli: Masson trichrome staining (a-d) and PAS staining (e-h) of kidney sections are shown for the treatment groups indicated at the top of the figure. Quantitation from PAS-stained sections showed that glomerular volume (i), mesangial matrix (j) and mesangial fraction (k) were all increased significantly by uninephrectomy in OVE mice but not in FVB mice. * indicates that OVE-uni values are greater than all other values and ${ }^{\#}$ indicates that OVE-sham values are greater than FVB-sham ( $\mathrm{p}<0.05$ by ANOVA). Nephrin mRNA



levels were reduced relative to FVB-sham ( $p<0.05$ by ANOVA, indicated by $\left.{ }^{\times}\right)$. OVE-uni nephrin mRNA levels were significantly lower than both FVB groups $(\mathrm{p}<0.05$ by ANOVA, indicated by $^{+}$). Micrographs and quantitation were obtained from at least 4 mice for each treatment. Methods for measurement of glomerular volume, mesangial matrix expansion and nephrin mRNA expression are described in the Methods section. Orig. magnif. $\times 200$ for trichrome and $\times 400$ for PAS staining. 


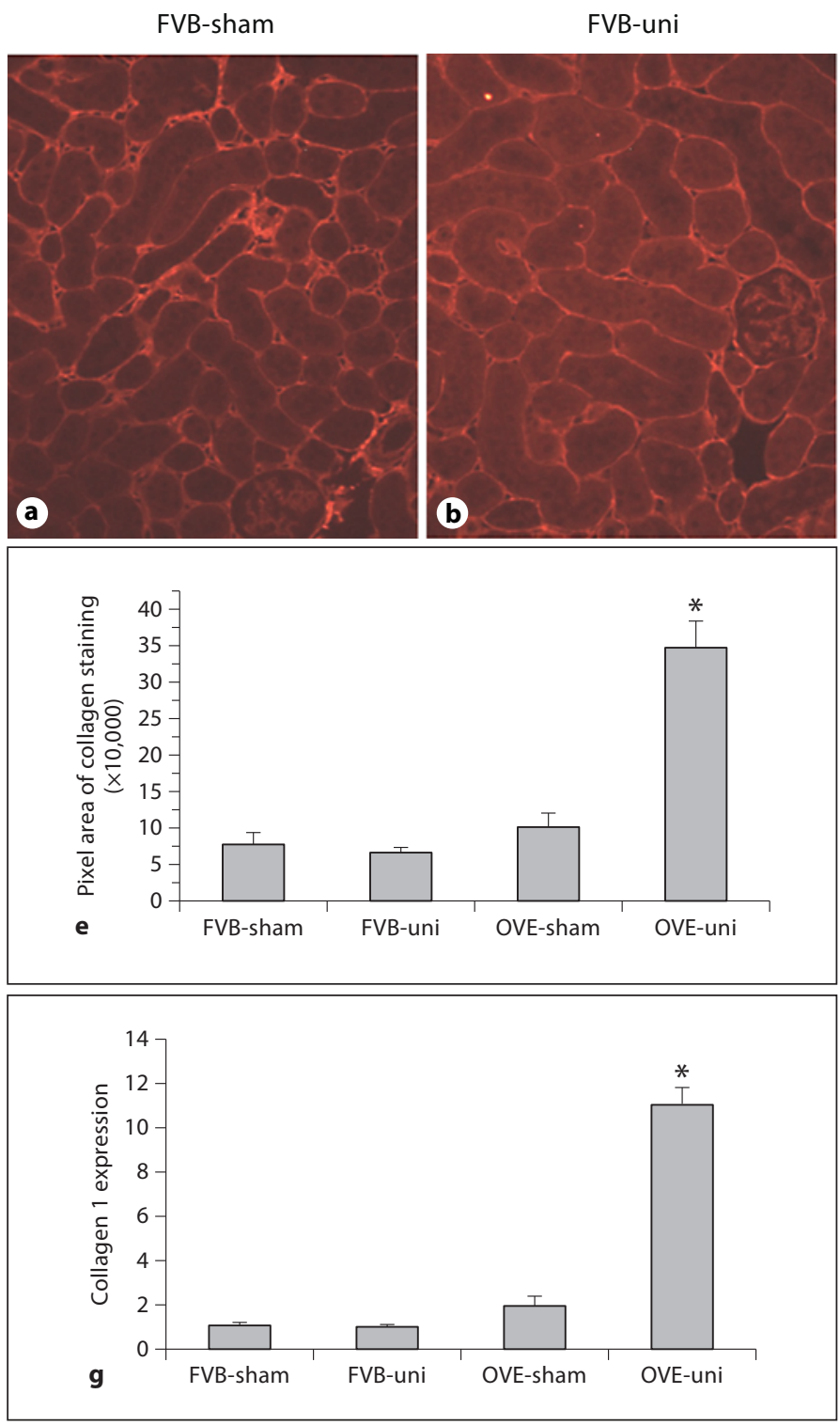

Fig. 5. In diabetic mice uninephrectomy produced an increase in the severity of fibrosis. Fibrosis was shown by collagen 1 staining (a-d). Renal fibrosis was quantitated (e) as the number of pixels stained for collagen per $200 \times$ field in the renal cortex, by procedures described in the Methods section. ${ }^{*}$ indicates that collagen deposition was greater in the OVE-uni group than in all other
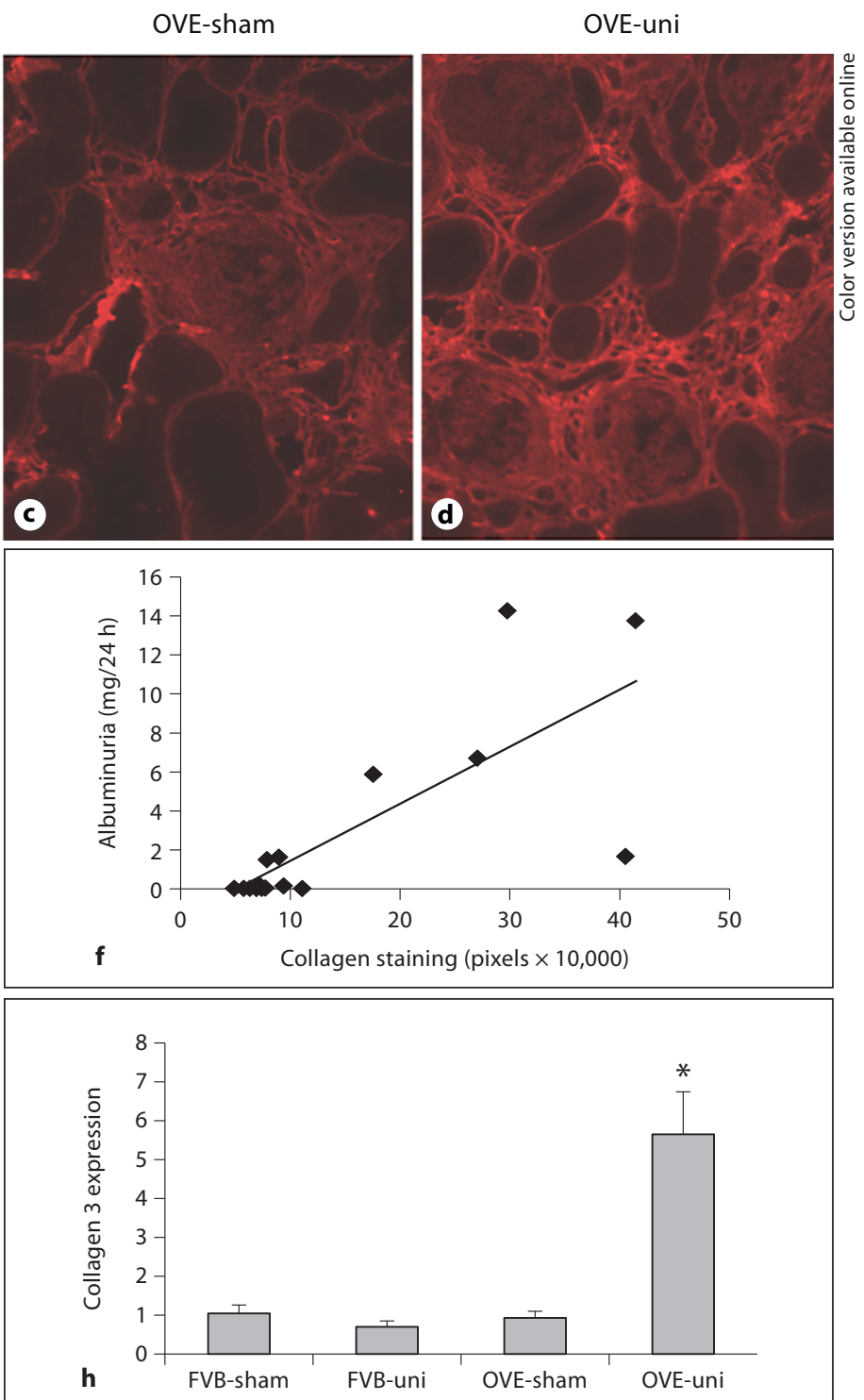

groups ( $\mathrm{p}<0.01$ by one-way ANOVA). $\mathbf{f}$ There was a significant correlation between the renal fibrosis score and the severity of albuminuria (Pearson correlation coefficient: 0.761, p < 0.0006). Levels of collagen 1 and collagen 3 mRNAs were significantly increased relative to all other groups ( $\mathrm{p}<0.01$ by one-way ANOVA).
(LAMA1); tissue metallopeptidase and their inhibitors MMP1, TIMP1 (tissue inhibitor of matrix metallopeptidase 1) and TIMP3; the adhesive molecules ICAM1 and VCAM1; the growth factors TGF- $\beta$, PDGF- $\beta$, HGF (hepatocyte growth factor), and the inflammatory and immune modulators chemokine ligand 2 (CCL2 or MCP-1), chemokine ligand 5 (CCL5 or RANTES), chemokine li- gand 3 (CXCL3), IL-10, CD14, CD40. In the UNI dataset, 26 genes were altered 2 -fold in this fibrosis pathway but only 9 genes were altered 2 -fold in the SHAM dataset. The higher representation of the UNI dataset is consistent with the greater fibrosis observed in OVE-uni kidneys compared to any other group. 
Fig. 6. Increased monocyte infiltration after uninephrectomy in OVE kidney. Staining for monocytes in FVB-sham (a), FVBuni (b), OVE-sham (c) and OVE-uni (d) kidney sections. The OVE-sham picture is from the only OVE-sham mouse that displayed positive monocyte staining. e Monocyte scores for all mice in each group. ${ }^{*}$ indicates that OVE-uni staining was greater than all other groups $(\mathrm{p}<0.05$ by one-way ANOVA).


Table 1. Comparison of uni and SHAM ratios derived from gene array and by rtPCR signals

\begin{tabular}{llcccc}
\hline Probe ID & Gene symbol & UNI array ratio & UNI rtPCR ratio & SHAM array ratio & SHAM rtPCR ratio \\
\hline 1423954 & C3 & 10.29 & $11.37 \pm 2.91^{*}$ & 3.71 & $2.29 \pm 0.31^{*}$ \\
1418126 & CCL5 & 5.51 & $8.12 \pm 4.01$ & 2.89 & $1.16 \pm 0.27$ \\
1460227 & TIMP1 & 99 & $74.1 \pm 14.13^{* *}$ & $-^{\#}$ & $4.63 \pm 1.94$ \\
1419089 & TIMP3 & 1.11 & $2.12 \pm 0.66$ & 1.12 & $0.79 \pm 0.07$ \\
1417256 & MMP13 & 0.96 & $0.44 \pm 0.08^{* *}$ & 0.47 & $0.57 \pm 0.22^{*}$ \\
1448162 & VCAM1 & 4.66 & $7.61 \pm 2.13^{*}$ & 0.74 & $0.64 \pm 0.07^{*}$ \\
1422142 & nephrin & 0.36 & $0.53^{* *}$ & 0.66 & $0.49^{*}$ \\
1423110 & collagen 1 & 6.48 & $10.83^{*}$ & 1.35 & 1.875 \\
1427884 & collagen 3 & 5.46 & $7.79^{*}$ & 0.86 \\
\hline
\end{tabular}

The UNI ratio was obtained by dividing OVE-uni by FVB-uni and the SHAM ratio was obtained by dividing OVE-sham by FVBsham. For rtPCR, 4 samples were used per group. For gene array, the same 4 RNA samples were pooled to prepare one probe per group.

${ }^{*} \mathrm{p}<0.05,{ }^{* *} \mathrm{p}<0.01$ for OVE versus FVB. ${ }^{\#}$ Both OVE and FVB values were reported as absent on gene array. 
Table 2. The top 15 canonical pathways significantly altered in OVE-uni kidneys compared to FVB-uni kidneys as identified by Ingenuity Pathway Analysis program

\begin{tabular}{lllll}
\hline $\begin{array}{l}\text { Ingenuity } \\
\text { canonical pathway }\end{array}$ & $\begin{array}{l}\text { p value } \\
\text { UNI } \\
\text { dataset }\end{array}$ & $\begin{array}{l}\text { Total } \\
\text { genes in }\end{array}$ & $\begin{array}{l}\text { Genes } \\
\text { pathway }\end{array}$ & Genes in Genes altered at least twofold in the UNI dataset and their fold change (OVE/FVB) \\
& &
\end{tabular}

\begin{tabular}{|c|c|c|c|c|c|}
\hline $\begin{array}{l}\text { Hepatic fibrosis/ } \\
\text { hepatic stellate } \\
\text { cell activation }\end{array}$ & $2.0 \mathrm{E}-06$ & 135 & 26 & 9 & $\begin{array}{l}\text { AGT (2.3), CCL2 (7.5), CCL5 (5.5), CD14 (5.3), CD40 (37.7), COL1A1 (5.2), COL1A2 (6.5), } \\
\text { COL3A1 (5.5), CXCL3 (9.6), ECE1 (0.48), FAS (0.23), FN1 (2.3), HGF (15.5), ICAM1 (2.0), } \\
\text { IL-10 (2.8), LAMA1 (2.1), LBP (2.8), MMP1 (0.21), MYH1 (2.3), PDGFB (2.3), STAT1 } \\
\text { (0.42), TGFB1 (5.8), TGFB2 (2.2), TIMP1 (99.0), TIMP2 (0.38), VCAM1 (5.9) }\end{array}$ \\
\hline $\begin{array}{l}\text { Coagulation } \\
\text { system }\end{array}$ & $4.0 \mathrm{E}-05$ & 37 & 11 & 0 & $\begin{array}{l}\text { BDKRB1 (0.43), F3 (2.41), F5 (0.42), F13A1 (2.6), F2R (4.7), FGA (2.5), FGG (6.1), KLKB1 } \\
\text { (0.48), SERPINA1 (14.3), SERPINC1 (0.48), SERPINE1 (3.9) }\end{array}$ \\
\hline $\begin{array}{l}\text { Dendritic cell } \\
\text { maturation }\end{array}$ & 0.0001 & 165 & 23 & 15 & $\begin{array}{l}\text { AKT3 (0.30), BCL3 (2.5), CD40 (37.7), CD86 (23.7), CD1D (2.4), COL1A1 (5.2), COL1A2 } \\
\text { (6.5), COL3A1 (5.5), FSCN1 (2.0), HLA-DMB (2.5), ICAM1 (2.0), IFNA5 (0.02), IL-10 } \\
\text { (2.8), IL-1F5 (0.40), IL-1F6 (3.1), IL-1F8 (0.24), MAPK13 (2.5), NFKBIE (2.7), PIK3R5 } \\
\text { (2.4), STAT1 (0.42), STAT2 (0.50), TLR2 (2.18), TYROBP (2.7) }\end{array}$ \\
\hline
\end{tabular}

Leukocyte $\quad 0.0003 \quad 195 \quad 27 \quad 12 \quad$ ACTG2 (2.1), ARHGAP4 (0.42), ARHGAP6 (0.48), ARHGAP9 (6.4), CD44 (6.4), CLDN3
extravasation $\quad$ (4.3), CLDN23 (2.2), CYBB (2.6), F11R (8.0), ICAM1 (2.0), ITGB1 (2.1), MAPK13 (2.5), signaling $\quad$ MMP12 (5.4), MMP14 (4.4), MMP1 (0.21), MSN (3.2), NCF2 (2.7), PIK3R5 (2.4), PRKCZ (0.42), PRKD1 (0.48), RAC1 (0.26), RHOA (0.29), TIMP1 (99.0), TIMP2 (0.38), VAV2 (0.36), VCAM1 (5.9)

\begin{tabular}{|c|c|c|c|c|c|}
\hline $\begin{array}{l}\text { Acute phase } \\
\text { response } \\
\text { signaling }\end{array}$ & 0.0004 & 178 & 26 & 17 & $\begin{array}{l}\text { AGT (2.3), AKT3 (0.31), ALB (0.31), BCL3 (2.5), C3 (10.3), CP (4.2), FGA (2.5), FGG (6.1), } \\
\text { FN1 (2.3), HPX (0.43), IL-1F5 (0.40), IL-1F6 (3.1), IL-1F8 (0.24), KLKB1 (0.48), LBP ( } 2.8) \text {, } \\
\text { MAPK13 (2.5), NFKBIE (2.7), RBP1 (2.0), SAA2 (256.9), SEROINA1 (14.3), SERPINA3 } \\
\text { (7.7), SERPINE1 (3.9), SOCS3 (8.1), SOCS4 (2.9), STAT3 (0.50), TTR (0.43) }\end{array}$ \\
\hline
\end{tabular}

Cell cycle: G2/M $\quad 0.0006 \quad 43 \quad 10 \quad 4 \quad$ BRCA1 (9.3), CCNB1 (2.5), CNNB2 (2.4), CDC2 (44.9), CDKN1A (6.4), CHEK1 (4.4),
DNA damage $\quad$ KAT2B (0.40), MDM2 (2.2), TOP2A (3.5), UBD (8.7)

checkpoint regulation

\begin{tabular}{|c|c|c|c|c|c|}
\hline IL-10 signaling & 0.0006 & 71 & 13 & 11 & ARG2 (2.0), BCL3 (2.5), CCR1 (2.7), CD14 (5.3) \\
\hline
\end{tabular}

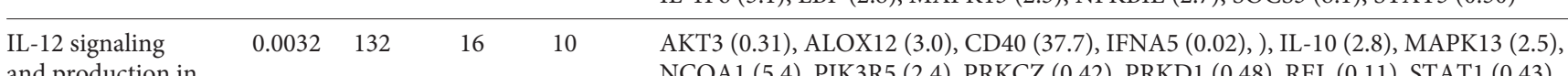
and production in $\quad$ NCOA1 (5.4), PIK3R5 (2.4), PRKCZ (0.42), PRKD1 (0.48), REL (0.11), STAT1 (0.43), macrophages $\quad$ TGFB1 (5.8), TGFB2 (2.2), TLR2 (2.2), ZNF668 (0.40)

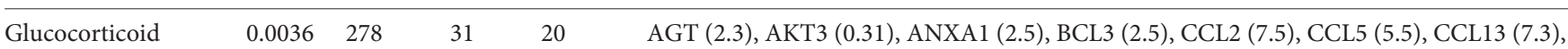
receptor signaling CDKN1A (6.4), CSN2 (0.23), CXCL3 (9.6), ESR1 (0.19), FGG (6.1), HSPA9 (5.4), ICAM1 (2.0), IL-10 (2.8), KAT2B (0.40), MAPK13 (2.5), MED14 (0.48), MMP1 (0.21), NCOA1 (5.4), NFKBIE (2.7), PCK1 (0.38), PCK2 (0.50), PIK3R5 (2.4), RAC1 (0.26), SERPINE1 (3.9), STAT1 (0.42), STAT3 (0.50), TGFB1 (5.8), TGFB2 (2.2), VCAM1 (5.9)

\begin{tabular}{|c|c|c|c|c|c|}
\hline TREM1 signaling & 0.0071 & 69 & 10 & 0 & $\begin{array}{l}\text { AKT3 (0.30), CCL2 (7.5), CD40 (37.7), CD86 (23.7), ICAM1 (2.0), IL-10 (2.8), ITGB1 (2.1), } \\
\text { STAT3 (0.50), TLR2 (2.2), TYROBP (2.7) }\end{array}$ \\
\hline $\begin{array}{l}\text { Histidine } \\
\text { metabolism }\end{array}$ & 0.0162 & 111 & 8 & 8 & $\begin{array}{l}\text { ABP1 (4.7), ALDH1A1 (2.0), ALDH1A7 (3.8), ALDH1L1 (0.42), AOC3 (3.6), CNDP1 } \\
\text { (0.41), FTCD (2.1), HDC (4.4) }\end{array}$ \\
\hline $\begin{array}{l}\text { Aryl hydrocarbon } \\
\text { receptor signaling }\end{array}$ & 0.0166 & 155 & 18 & 12 & $\begin{array}{l}\text { ALDH18A1 (3.4), ALDHA1 (2.0), ALDH1L1 (0.42), CCNA2 (70.2), CCNE1 (4.2), CCNE2 } \\
\text { (3.0), CDKN1A (6.4), CHEK1 (4.4), CYP1A2 (4.9), CYP1B1 (2.1), ESR1 (0.19), MDM2 } \\
\text { (2.2), MYC (2.3), NFIC (0.37), SLC35A2 (3.1), TGFB1 (5.8), TGFB2 (2.2), TRIP11 (0.23) }\end{array}$ \\
\hline $\begin{array}{l}\text { Xenobiotic } \\
\text { metabolism } \\
\text { signaling }\end{array}$ & 0.0178 & 254 & 26 & 19 & $\begin{array}{l}\text { ABCB1 (0.32), ALDH18A1 (3.4), ALDH1A1 (2.0), ALDH1L1 (0.42), CAMK4 (3.0), CES7 } \\
\text { (0.30), CES1 (0.37), CHST11 (2.0), CYP1A2 (4.8), CYP1B1 (2.1), CYP2C8 (0.43), ES22 } \\
\text { (0.38), FMO2 (0.45), GRIP1 (2.3), HS3ST1 (2.4), HS3ST3B1 (0.48), MAPK13 (2.5), NCOA1 } \\
\text { (5.4), PIK3R5 (2.4), PPM1J (5.1), PPP2R1B (0.40), PRKCZ (0.42), PRKD1 (0.48), UGT2B17 } \\
\text { (12.1), UGT2B35 (0.32), UST (0.45) }\end{array}$ \\
\hline
\end{tabular}

\begin{tabular}{lllllll}
\hline $\begin{array}{l}\text { Hepatic } \\
\text { cholestasis }\end{array}$ & 0.02 & 166 & 17 & 15 & $\begin{array}{l}\text { ABCB1 (0.32), ABCG8 (0.32), ADCY7 (2.7), BCL3 (2.5), CD14 (5.3), CYP7A1 (0.43), ESR1 } \\
\text { (0.19), GCGR (0.43), IL-1F5 (0.40), IL-1F6 (3.1), IL-1F8 (0.24), LBP(2.8), INSR (0.34), }\end{array}$ \\
& & & & & & NFKBIE (2.7), PRKAR1B (2.3), PRKCZ (0.42), PRKD1 (0.48)
\end{tabular}

Pathways are ordered by $\mathrm{p}$ value, shown in the second column. The number of genes in the pathway altered twofold in the UNI and SHAM datasets are shown in the fourth and fifth columns, respectively. The last column lists the symbol and fold change for each gene in the UNI dataset. 


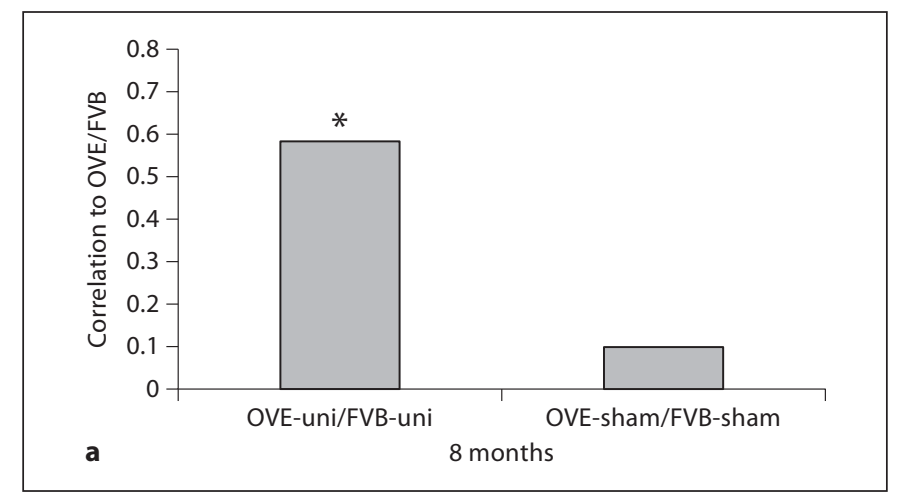

Fig. 7. Changes in gene expression of untreated OVE mice at 4 and 8 months of age [manuscript submitted] correlated to changes in gene expression of OVE-uni mice and OVE-sham mice. a Untreated OVE results obtained at 8 months were most closely correlated to OVE-uni results. b At 4 months of age, OVE results were

Several inflammatory- and immune-related canonical pathways were also shown to be significantly changed in the UNI dataset. These included leukocyte extravasation signaling, acute phase response signaling, IL-10, IL-12 signaling, the complement system and dendritic cell maturation. For each of these pathways, more genes were upregulated by diabetes in the UNI dataset than in the SHAM dataset.

The current results were compared to a separate microarray data set obtained previously from untreated OVE diabetic mice and control FVB mice at 4 and 8 months of age (http://www.ncbi.nlm.nih.gov/gds?term = ove26 [manuscript submitted]). Figure $7 \mathrm{a}$ and $\mathrm{b}$ shows that the UNI dataset was more closely correlated to the previous 8 months' results, while the SHAM dataset was more closely correlated to the prior 4 months' results. These data indicate that uninephrectomy accelerated DN not only at the histopathology level, but also at the level of gene expression.

\section{Discussion}

In this report we describe a faster, more advanced model of DN by application of uninephrectomy. The impact of uninephrectomy was minimal in non-diabetic mice but severe in OVE mice. Uninephrectomy greatly accelerated the progression of OVE DN with respect to albuminuria, mesangial matrix expansion, interstitial fibrosis and gene expression.

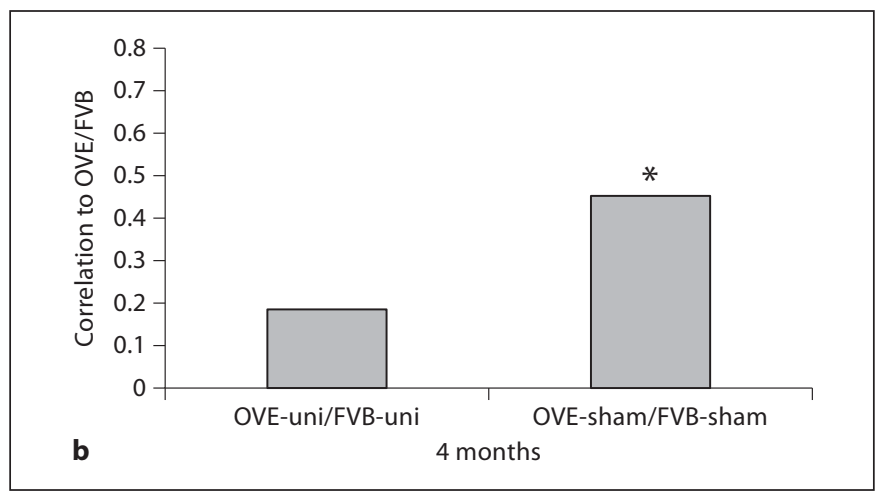

most closely correlated to OVE-sham results. ${ }^{*}$ indicates that $\mathrm{p}$ values of Pearson product moment correlations were $<0.01$. The genes used to compute the correlations were those altered up or down 2-fold in OVE compared to FVB.

Uninephrectomy Dramatically Increased Albuminuria in OVE Mice

Uninephrectomy was performed at 2 months of age. At this time, OVE mice had already started to develop albuminuria and increased GFR. Uninephrectomy rapidly increased albuminuria in OVE mice within 1 week of surgery. This elevation of albuminuria in nephrectomized diabetic mice was highly progressive during the 10 -week follow-up. At the end of the 10 weeks, albuminuria in OVE-uni mice was 600 times higher than in nondiabetic mice and 6 times higher than albuminuria in OVE-sham mice. In FVB mice, uninephrectomy had no effect on albuminuria. Uninephrectomy appears to act synergistically with diabetes to increase glomerular leakage and/or reduce tubular albumin reabsorption. Compared with other diabetic mice treated with uninephrectomy [8-10], the albuminuria changes in OVE-uni mice are enormous. The very high levels of albuminuria that develop with uninephrectomy should be advantageous both to analyze the mechanisms producing albuminuria and to study the secondary damage that occurs in the tubules as a result of albumin exposure.

\section{GFR Response to Uninephrectomy in OVE Mice}

In our previous study, GFR, measured by inulin clearance, did not decline significantly until OVE mice reached 9 months [6]. At this age, OVE GFR was modestly (20\%) but significantly reduced compared to normal mice. In the current study, the estimated GFR of OVE-uni mice declined within 10 weeks of uninephrectomy, which is 4.5 months of age. Endogenous creatinine clearance was used to estimate GFR. Although HPLC measurement of 
creatinine has been adopted by the Animal Models of Diabetic Complications Consortium (AMDCC) as the preferred method to measure plasma creatinine in mice [17], creatinine clearance does not take into account the effect of proximal tubular creatinine secretion, which was recently reported to account for $30-50 \%$ of total urine creatinine in mice [18]. In addition, while the GFR results with OVE mice suggest that uninephrectomy accelerated the drop in GFR, it needs to be recognized that the GFR of OVE-uni mice was not significantly lower than GFR in FVB-sham mice. Clearly, OVE-uni mice do not reach the criteria of a $50 \%$ reduction in GFR as laid down by the AMDCC [5]. Therefore it cannot be concluded yet that OVE-uni mice provide a model of impaired GFR

Increased Renal Hypertrophy, Fibrosis and Monocyte Infiltration in Uninephrectomized OVE Mice

Uninephrectomy had a striking effect on morphology of the remnant kidney in OVE mice. Ten weeks after surgery, the kidney size in OVE-uni mice almost doubled. This dramatic renal hypertrophy was consistent with the increase in glomerular size as well as hypertrophy and dilation of renal tubules. Renal fibrosis in OVE-uni mice was much more severe than in OVE-sham mice at the same age and even more extensive than our previous observations $[6,19]$ of fibrosis in OVE mice at age 8-9 months. At 4.5 months of age, infiltrating monocytes were common in OVE-uni kidneys but rare in OVEsham kidneys. This is consistent with our previous observation of increasing infiltrating cells with age in OVE mice. Overall, the main features of pathology, tubulointerstitial fibrosis and inflammatory cell infiltration, were similar in OVE-uni mice and 8- to 9-month-old OVE mice, which indicates that the uninephrectomy did not create new pathologic features but accelerated the development of DN in OVE-uni mice.

\section{Changes of Profibrotic and Inflammatory Gene}

Expression in OVE after Uninephrectomy

The accelerated progression of renal damage, especially renal fibrosis and inflammation in OVE-uni mice, was also identified at the gene expression level. The use of pooled samples rather than multiple individual samples prevents the use of these results for identification of statistically significant changes in expression of individual genes. However, treatment comparisons can be made for pathway analysis in pathways that contain tens or hundreds of different genes. The hepatic fibrosis/hepatic stellate cell activation pathway is the Ingenuity canonical pathway that is most closely related to kidney fibrosis.
This pathway was by far the most significant ( $\mathrm{p}=$ 0.000002) pathway identified by gene array in OVE-uni mice. The increased expression of fibrosis-related genes coincided with the dramatically increased renal fibrosis in OVE-uni mice, which indicates correspondence between gene array and kidney pathology. Furthermore, when global gene expression profiles in OVE-uni kidneys were compared with gene expression profiles of untreated OVE mice at 4 and 8 months of age, the highest correlation was with 8-month-old OVE mice. This provides additional support for the concept that uninephrectomy accelerates the development of DN in OVE mice.

The majority of the top pathways upregulated in OVEuni kidneys were inflammatory- and immune-related, including acute phase response signaling, leukocyte extravasation, IL-6, IL-10, IL-12 signaling, TREM1 signaling, dendritic cell maturation and the complement system. This upregulation of inflammatory pathways is consistent with the known importance of inflammation in progression of DN and chronic renal diseases [20-24] and suggests that more rapid activation of these pathways may be involved in the accelerated renal pathology in OVE-uni mice. The upregulation of inflammatory cell gene expression in OVE-uni kidneys coincides closely with the influx of inflammatory cells in these kidneys.

Uninephrectomy clearly had a synergistic effect with diabetes since all parameters changed dramatically after surgery in OVE mice but modestly or not at all in nondiabetic mice. Uninephrectomy produces renal damage by increasing intraglomerular pressure and single nephron filtration rate $[8,10]$. This may exacerbate the already damaged glomerular filtration barrier of OVE mice resulting in a tremendous increase in albumin leaking into tubules of OVE-uni mice. Albuminuria is thought to activate secretion of inflammatory and profibrotic mediators from proximal tubule cells [25-27]. If this is correct, then the very extensive fibrotic and inflammatory response produced by uninephrectomy of OVE mice was probably initiated by increased albumin leakage from the glomerulus.

A caveat for the OVE-uni model is that the mechanism and pathology of uninephrectomy-accelerated nephropathy may be different from DN. Uninephrectomy causes renal damage via abnormal renal hemodynamics in the remaining kidney. However, it is not certain that this mechanism plays a major role in human DN. Also, the extent of segmental glomerulosclerosis in OVE-mice was more than typically observed in DN. Uncertainties about the mechanism of the OVE-uni model should be weighed against the advantages of speed and severity of nephropathy in this model. 


\section{Conclusion}

Uninephrectomy dramatically exacerbated DN in OVE mice but had only modest effects on FVB mice. Albuminuria, fibrosis, mesangial matrix expansion, monocyte infiltration and altered gene expression were all as advanced in 4.5-month-old OVE-uni mice as in untreated OVE mice at 8 months of age. The 10 -week period after surgery provides a valuable window to analyze large changes in diabetic pathology over a short period of time and to test the efficacy of new therapeutic agents against DN.

\section{Acknowledgements}

S.Z. was supported by National Institutes of Health grant T32HL076138 from the National Heart, Lung, and Blood Institute. This work was supported by grants RO1DK072032 and Juvenile Diabetes Research Foundation grant 1-2005-88. We thank Sabina Waigel and Vennila Arumugam of the Cancer Center Microarray Core for probe hybridization and scanning and Yinlu Chen for help on microarray statistical analysis and Marcia Liu for the creatinine assay.

\section{References}

-1 Breyer MD, Qi Z, Tchekneva E: Diabetic nephropathy: leveraging mouse genetics. Curr Opin Nephrol Hypertens 2006;15:227-232.

$\checkmark 2$ Breyer MD: Stacking the deck for drug discovery in diabetic nephropathy: in search of an animal model. J Am Soc Nephrol 2008;19: 1623-1624.

-3 Nakagawa T, Sato W, Glushakova O, Heinig M, Clarke T, Campbell-Thompson M, Yuzawa Y, Atkinson MA, Johnson RJ, Croker B: Diabetic endothelial nitric oxide synthase knockout mice develop advanced diabetic nephropathy. J Am Soc Nephrol 2007;18: 539-550.

4 Kanetsuna Y, Takahashi K, Nagata M, Gannon MA, Breyer MD, Harris RC, Takahashi T: Deficiency of endothelial nitric oxide synthase confers susceptibility to diabetic nephropathy in nephropathy-resistant inbred mice. Am J Pathol 2007;170:1473-1484.

$\checkmark 5$ Brosius FC 3rd, Alpers CE, Bottinger EP, Breyer MD, Coffman TM, Gurley SB, Harris RC, Kakoki M, Kretzler M, Leiter EH, Levi M, McIndoe RA, Sharma K, Smithies O, Susztak K, Takahashi N, Takahashi T: Mouse models of diabetic nephropathy. J Am Soc Nephrol 2009;20:2503-2512.

6 Zheng S, Noonan WT, Metreveli NS, Coventry S, Kralik PM, Carlson EC, Epstein PN: Development of late-stage diabetic nephropathy in OVE26 diabetic mice. Diabetes 2004; 53:3248-3257.

7 Zheng S, Carlson EC, Yang L, Kralik PM, Huang Y, Epstein PN: Podocyte-specific overexpression of the antioxidant metallothionein reduces diabetic nephropathy. J Am Soc Nephrol 2008;19:2077-2085.

$\checkmark 8$ Levine DZ, Iacovitti M, Robertson SJ, Mokhtar GA: Modulation of single-nephron GFR in the $\mathrm{db} / \mathrm{db}$ mouse model of type 2 diabetes mellitus. Am J Physiol Regul Integr Comp Physiol 2006;290:R975-R981.
9 Ninichuk V, Kulkarni O, Clauss S, Anders HJ: Tubular atrophy, interstitial fibrosis, and inflammation in type 2 diabetic $\mathrm{db} / \mathrm{db}$ mice. An accelerated model of advanced diabetic nephropathy. Eur J Med Res 2007;12:351-355.

-10 Dworkin LD, Hostetter TH, Rennke HG Brenner BM: Hemodynamic basis for glomerular injury in rats with desoxycorticosterone-salt hypertension. J Clin Invest 1984; 73:1448-1461

11 Qi Z, Whitt I, Mehta A, Jin J, Zhao M, Harris RC, Fogo AB, Breyer MD: Serial determination of glomerular filtration rate in conscious mice using FITC-inulin clearance. AmJ Physiol Renal Physiol 2004;286:F590F596.

12 Dunn SR, Qi Z, Bottinger EP, Breyer MD, Sharma K: Utility of endogenous creatinine clearance as a measure of renal function in mice. Kidney Int 2004;65:1959-1967.

13 Hartner A, Eifert T, Haas CS, Tuysuz C, Hilgers KF, Reinhardt DP, Amann K: Characterization of the renal phenotype in a mouse model of Marfan syndrome. Virchows Arch 2004;445:382-388.

14 Sanden SK, Wiggins JE, Goyal M, Riggs LK, Wiggins RC: Evaluation of a thick and thin section method for estimation of podocyte number, glomerular volume, and glomerular volume per podocyte in rat kidney with Wilms' tumor-1 protein used as a podocyte nuclear marker. J Am Soc Nephrol 2003;14: 2484-2493.

15 Ziyadeh FN, Hoffman BB, Han DC, Iglesiasde la Cruz MC, Hong SW, Isono M, Chen S, McGowan TA, Sharma K: Long-term prevention of renal insufficiency, excess matrix gene expression, and glomerular mesangial matrix expansion by treatment with monoclonal antitransforming growth factor- $\beta$ antibody in $\mathrm{db} / \mathrm{db}$ diabetic mice. Proc Natl Acad Sci USA 2000;97:8015-8020.

$\checkmark 16$ Chen S, Hong SW, Iglesias-de la Cruz MC, Isono M, Casaretto A, Ziyadeh FN: The key role of the transforming growth factor- $\beta$ system in the pathogenesis of diabetic nephropathy. Ren Fail 2001;23:471-481.
17 Qi Z, Breyer MD: Measurement of glomerular filtration rate in conscious mice. Methods Mol Biol 2009;466:61-72.

18 Eisner C, Faulhaber-Walter R, Wang Y, Leelahavanichkul A, Yuen PS, Mizel D, Star RA, Briggs JP, Levine M, Schnermann J: Major contribution of tubular secretion to creatinine clearance in mice. Kidney Int 2010; 77:519-526.

$\checkmark 19$ Powell DW, Bertram CC, Cummins TD, Barati MT, Zheng S, Epstein PN, Klein JB: Renal tubulointerstitial fibrosis in OVE26 type 1 diabetic mice. Nephron Exp Nephrol 2009;111:e11-e19.

20 Vieyra MB, Heeger PS: Novel aspects of complement in kidney injury. Kidney Int 2010; 77:495-499.

21 Chow F, Ozols E, Nikolic-Paterson DJ, Atkins RC, Tesch GH: Macrophages in mouse type 2 diabetic nephropathy: correlation with diabetic state and progressive renal injury. Kidney Int 2004;65:116-128.

22 Chow FY, Nikolic-Paterson DJ, Atkins RC, Tesch GH: Macrophages in streptozotocininduced diabetic nephropathy: potential role in renal fibrosis. Nephrol Dial Transplant 2004;19:2987-2996.

23 Schmid H, Boucherot A, Yasuda Y, Henger A, Brunner B, Eichinger F, Nitsche A, Kiss E, Bleich M, Grone HJ, Nelson PJ, Schlondorff D, Cohen CD, Kretzler M: Modular activation of nuclear factor $-\kappa \mathrm{B}$ transcriptional programs in human diabetic nephropathy. Diabetes 2006;55:2993-3003.

24 Mora C, Navarro JF: Inflammation and diabetic nephropathy. Curr Diab Rep 2006;6: 463-468.

25 Christensen EI, Verroust PJ: Interstitial fibrosis: tubular hypothesis versus glomerular hypothesis. Kidney Int 2008;74:1233-1236.

26 Abbate M, Zoja C, Remuzzi G: How does proteinuria cause progressive renal damage? J Am Soc Nephrol 2006;17:2974-2984.

27 Wolf G, Ziyadeh FN: Cellular and molecular mechanisms of proteinuria in diabetic nephropathy. Nephron Physiol 2007;106:2631. 